\title{
56 Nichtinvasive Registrierung der fetalen Herzfrequenz
}

\author{
Nagel, J., Schaldach, M.
}

Zentralinstitut für Biomedizinische Technik der Universität Erlangen-Nürnberg

Die sichere Erfassung des fetalen Elektrokardiogramms (FEKG) zur Schlag-zu-Schlag Bestimmung der Herzfrequenz stellte bisher ein nicht zufriedenstellend gelöstes Problem fetaler Oberwachungssysteme dar. Erst unmittelbar vor der Geburt, nach Offnen der Fruchtblase, kann das FEKG mit Hilfe von Einschraubelektroden direkt vom Feten abgeleitet werden. Da diese Methode sich naturgemäß nicht während der Schwangerschaft anwenden läßt, muß dort auf die indirekte Ableitung vom Abdomen der Mutter zurückgegriffen werden. Die abdominalen elektrischen Signale weisen jedoch ein derart ungünstiges Signal/Störverhältnis auf, daß mit den Mitteln der bisher angewandten Filtertechniken weder ausreichende Information über die zeitlichen Veränderungen des FEKGs noch über die Mikrofluktuation der Herzfrequenz gewonnen werden konnte. Durch die Entwicklung neuer Signalverarbeitungsverfahren im Zentralinstitut wurde die Analyse des transabdominalen EKGs so weit verbessert, daß sie die für den klinischen Routineeinsatz erforderliche Zuverlässigkeit aufweist.

\section{MESSVERFAHREN}

Eine notwendige Voraussetzung zur sicheren Registrierung der fetalen Herztätigkeit ist die Abtrennung des im abdominal abgeleiteten Signal als Störung enthaltenen maternellen EKGs, bei der jedoch das FEKG

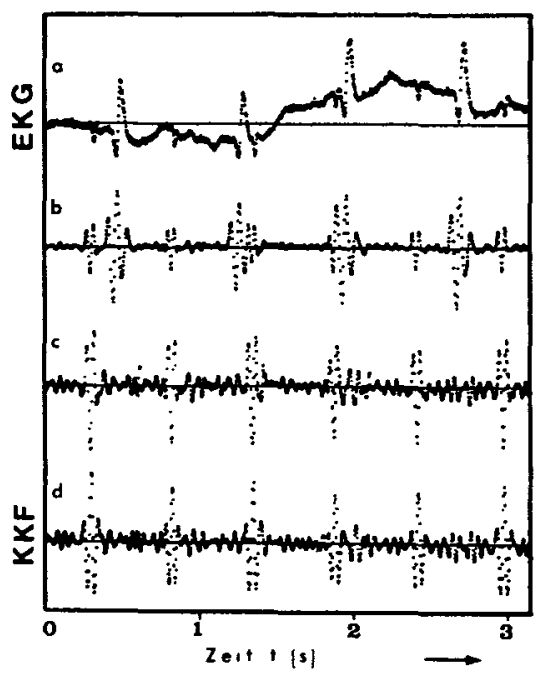

Abd. EKG

gefiltertes EKG

bereinigtes EKG

KKF

Abb. 1 Unterdrückung des maternellen EKGs im abdominal abgeleiteten Signalgemisch weder verfälscht noch unterdrückt werden darf. Unter Anwendung von Mittelwerttechniken konnte ein Verfahren realisiert werden, das die Trennung ermöglicht, ohne daß eine zusätzliche MEKG-Ableitung hierzu erforderlich ist (Abb. 1). Durch fortlaufende Mittelwertbildung wird ein Muster des im Signalgemisch enthaltenen MEKG gebildet. Durch Subtraktion dieses Musters vom abdominalen Signal wird das MEKG vollständig unterdrückt. Im Gegensatz zu herkömmlichen Verarbeitungsalgorithmen weist dieses Verfahren den besonderen Vorteil auf, daß auch diejenigen Teile des FEKG, die von maternellen QRSKomplexen überdeckt werden, einwandfrei registriert werden können.

Wegen der im abdominalen Signal verbleibenden Reststörungen ist die Zuverlässigkeit der Erkennung der fetalen QRS-Komplexe im bereinigten Signal mittels Schwellwertdetektoren noch nicht ausreichend für eine Schlag-zu-Schlag Registrierung der fetalen Herzfrequenz. Das Signal wird deshalb zusätzlich einer Korrelationsanalyse unterworfen, deren maßgeblicher Vorteil darin besteht, daß die fetalen QRS-Komplexe auch dann noch erkannt werden können, wenn ihre Amplitude kleiner als die von Störimpulsen ist. Darüber hinaus werden durch die

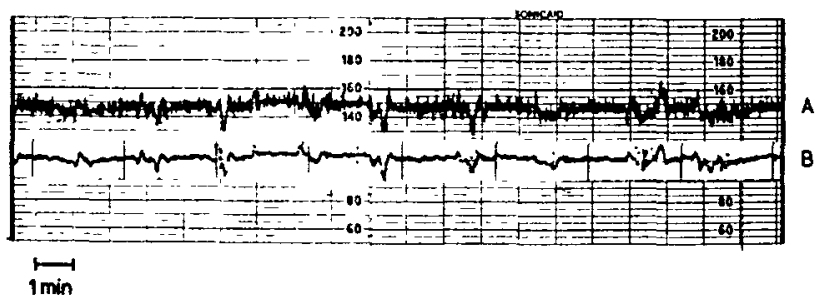

Abb. 2 Fetale Herzfrequenzkurve, aus Ultraschalldopplersignal (A) und transabdominalem EKG (B) berechnet

Anwendung der Korrelationsanalyse Triggerfehler vermieden, die bei herkömmlichen Verfahren unvermeidbar sind, wenn die Flanken der QRS-Komplexe durch Störsignale verfälscht werden. Klinische Tests zeigten eine Steigerung der Entdeckungsquote für das fetale EKG von bisher maximal $50 \%$ auf $90 \%$.

\section{ERFAHRUNGEN}

Die bisher gesammelten klinischen Erfahrungen mit einem nach dem beschriebenen Verfahren arbeitenden Gerätesystem zeigten, daß es den bekannten Kardiotokographen weit überlegen ist. Dies betrifft insbesondere die Zuverlässigkeit in der Registrierung des EKGs auch unter der Geburt sowie die Schlag-zu-Schlag-Bestimmung der fetalen Herzfrequenz mit einer großen Genauigkeit.

Zum Nachweis der Effektivität des Verfahrens wurden vergleichende Untersuchungen mit den bisher bekannten Systemen durchgeführt. 


\section{Ultraschall- und Phonokardiographie}

Die bisher gebräuchlichsten Methoden zur Registrierung der fetalen Herzfrequenz (FHF) sind die Ultraschall- und Phonokardiographie. Sie weisen jedoch wesentliche Nachteile auf:

- Aufgrund der Wellenformschwankungen der registrierten Signale treten beachtliche Triggerfehler (Jitter) auf, die nichtvorhandene Kurzzeitschwankungen der FHF vortäuschen (Ább. 2).

- Bei Kindsbewegungen und Lageveränderungen der Mutter müssen die Signalaufnehmer meist neu positioniert werden.

- Sehr geringe Zuverlässigkeit bei adipösen Schwangeren.

Diese Nachteile konnten mit Hilfe des neuen Analyseverfahrens überwunden werden.

\section{Abdominales Elektrokardiogramm}

Die bisher verfügbaren Geräte zur Verarbeitung des transabdominalen EKGs werden wegen ihrer geringen Zuverlässigkeit in der Praxis nur selten eingesetzt. Da sie das fetale und maternelle EKG nicht separieren können, bleibt wegen der häufigen Koinzidenz der R-Zacken ein großer Teil der fetalen QRS-Komplexe (oft > $50 \%$ ) prinzipiell unerkennbar. Darüber hinaus ist die ausschließlich benutzte Schwellwertanalyse des FEKG wegen des ungünstigen Signal/Störverhältnisses des transabdominalen FEKGs sehr unzuverlässig. Dementsprechend ist eine präzise Erfassung der instantanen FHF kaum möglich (Abb. 3).

Demgegenüber konnten bei dem neuen Verfahren diese Fehlerquellen durch die Subtraktion des MEKGs und die nachfolgende Korrelationsanalyse ausgeschaltet werden.

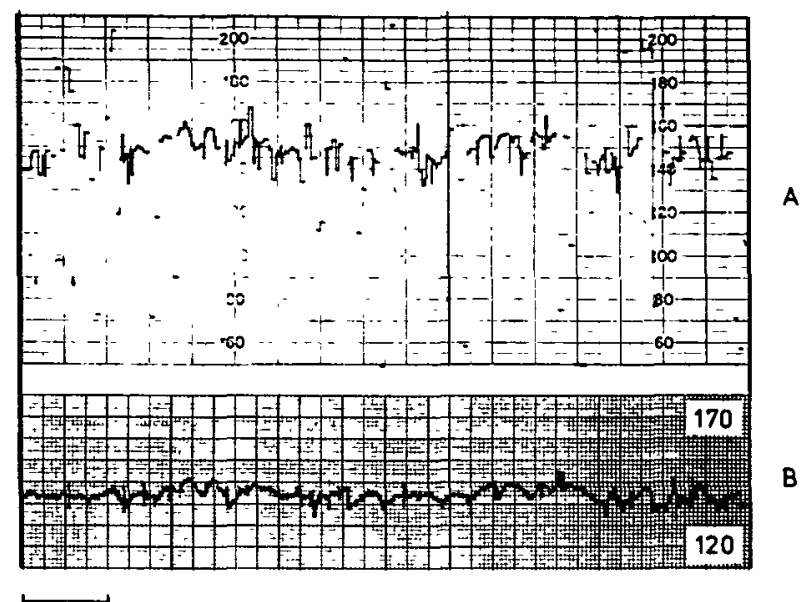

1 min

Abb. 3 FHF, abgeleitet aus dem Transabdominalen EKG; (A) mit herkömmlichem Verfahren, (B) mit Subtraktionsund Korrelationsalgorithmus

Insgesamt zeigen die Erfahrungen aus den klinischen Untersuchungen, daß das im Zentralinstitut entwickelte Verfahren zur transabdominalen Erfassung des fetalen Elektrokardiotokogramms zur Verbesserung der Uberwachung von Schwangerschaft und Geburt einen wesentlichen Beitrag liefern kann.

A

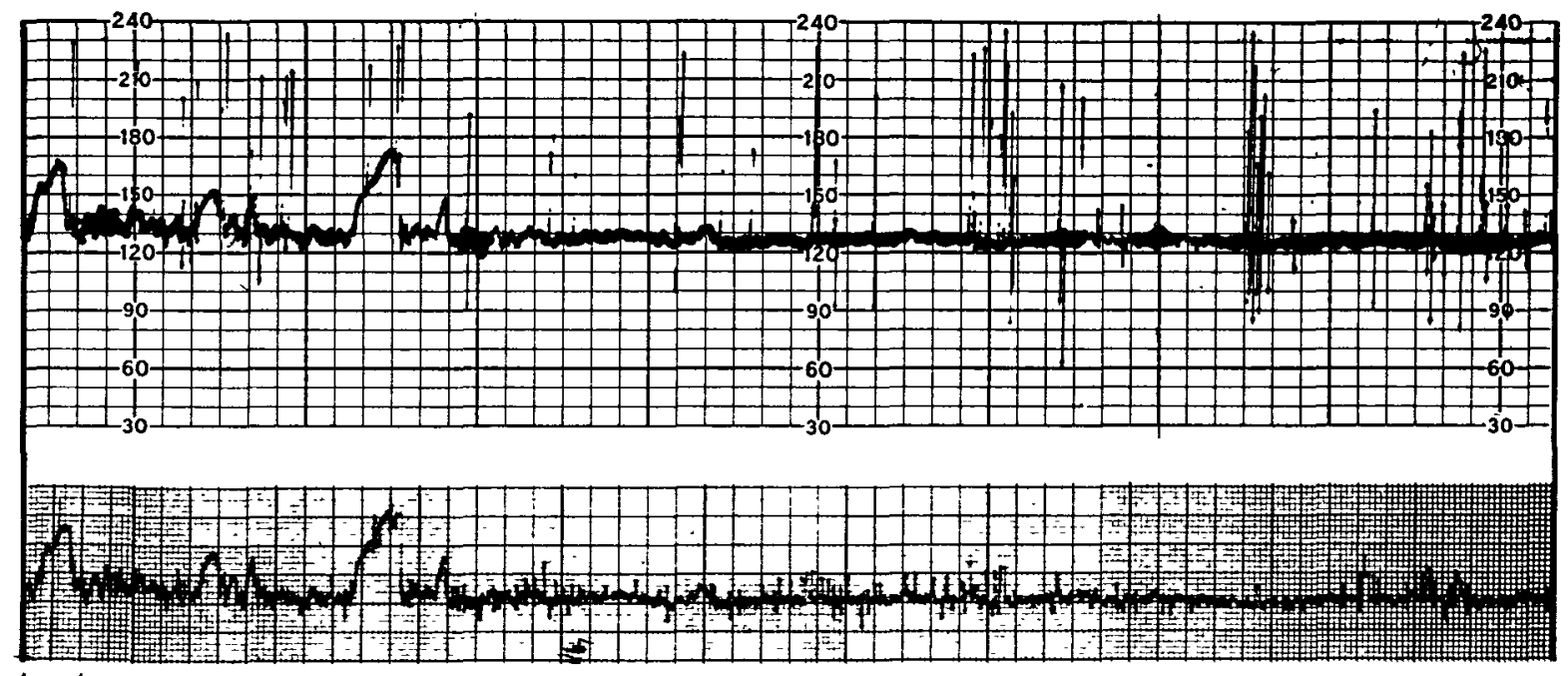

$\longmapsto-1$

$1 \mathrm{~min}$

Abb. 4 FHF, mit Scalp-Elektrode (A) und transabdominal (B) registriert

\section{Direkt abgeleitetes fetales Elektrokardiogramm}

Nach dem Óffnen der Fruchtblase kann das FEKG direkt vom Feten abgeleitet werden. Während von medizinischer Seite vermehrt Bedenken gegen die Applikation von Kopfschwartenelektroden erhoben werden, ist das direkte fetale Elektrokardiogramm meßtechnisch gesehen das am besten geeignete Signal zur Erfassung der FHF. Aus ihm kann die Herzfrequenz auch mit herkömmlichen Methoden relativ störungsfrei ermittelt werden. Die Beurteilung der Qualität eines Verfahrens zur Registrierung der fetalen Herzaktion sollte daher stets auch den Vergleich mit dem direkten FEKG umfassen. Abb. 4 zeigt eine derartige Messung. In beiden Kurven ist die gleiche Information enthalten. Die Fehlerquote bei der Registrierung des transabdominalen EKGs beträgt nur etwa $1.2 \%$.

Biomedizinische Technik Band 23

\section{LITERATUR}

1. Bemmel, van J.H.

"Detection and processing of foetal electrocardiograms"

Institute of Medical Physics TNO, Utrecht

Report 1.10.49-1 October 1969

2. Fischer, W.M.

"Kardiotokographie"

Georg Thieme Verlag Stuttgart (1973)

3. Nagel, J., Thull, R., Schaldach, M.

"Die Korrelationstechnik zur Überwachung fetaler Vitalfunktionen" Biomedizinische Technik Band 20, Mai 1975

4. Sureau, C. et coll.

"Interet actuel de l'electrocardiographie foetale"

Gyn. Obst., Paris, 1968, t.67, $n^{\circ} 2$ 